\title{
DAICS: A Deep Learning Solution for Anomaly Detection in Industrial Control Systems
}

\author{
Maged Abdelaty ${ }^{\alpha \beta}$, Roberto Doriguzzi-Corin ${ }^{\alpha}$, Domenico Siracusa $^{\alpha}$ \\ ${ }^{\alpha}$ ICT, Fondazione Bruno Kessler - Italy \\ ${ }^{\beta}$ University of Trento - Italy
}

\begin{abstract}
Deep Learning is emerging as an effective technique to detect sophisticated cyber-attacks targeting Industrial Control Systems (ICSs). The conventional approach to detection in literature is to learn the "normal" behaviour of the system, to be then able to label noteworthy deviations from it as anomalies. However, during operations, ICSs inevitably and continuously evolve their behaviour, due to e.g., replacement of devices, workflow modifications, or other reasons. As a consequence, the accuracy of the anomaly detection process may be dramatically affected with a considerable amount of false alarms being generated. This paper presents DAICS, a novel deep learning framework with a modular design to fit in large ICSs. The key component of the framework is a 2-branch neural network that learns the changes in the ICS behaviour with a small number of data samples and a few gradient updates. This is supported by an automatic tuning mechanism of the detection threshold that takes into account the changes in the prediction error under normal operating conditions. In this regard, no specialised human intervention is needed to update the other parameters of the system. DAICS has been evaluated using publicly available datasets and shows an increased detection rate and accuracy compared to state of the art approaches, as well as higher robustness to additive noise.
\end{abstract}

Index Terms-Anomaly detection, Domain shift, Deep Learning, Industrial control networks

\section{INTRODUCTION}

Nefarious cyber-attacks targeting Industrial Control Systems (ICSs) may cause service downtime or material losses in industrial production sites, with potential negative repercussions on the lives of citizens. A notable example is the blackout attack against the Ukrainian power grid perpetrated in late 2015 [1], which exploited the BlackEnergy (BE) malware. In that occasion, the attackers intruded remotely into the computers of three regional power distribution companies in a coordinated manner. They executed a malicious code to alter the firmware of specific control devices and to instruct unscheduled disconnections from servers. The attack affected thousands of users and left them without electricity. Another example is the attack the Iranian nuclear program [2] in 2010 by means of the Stuxnet work. The worm infected the code running inside the Programmable Logic Controllers (PLCs), collecting information on the ICS and damaging the centrifuges inside the plant by repeatedly changing their rotation speed.

The increasing occurrence of such attacks and their complexity have motivated the development of intrusion detection solutions for ICSs based on machine learning techniques. Among the different approaches proposed in the scientific literature, a popular and powerful technique is the one-class classification. At the training stage, solutions based on one-class classification build a model that represents the normal behaviour of the ICS (the class of "normality"). At the production stage, the detection system uses that model to verify whether the behaviour of the live system matches the expected normal behaviour. Deviations from the normality, usually determined through thresholds on the classification error, are flagged as anomalies.

These algorithms can be very sensitive to abnormal behaviours, including zero-days anomalies or attacks and faulty devices or sensors, since they may evade the detection process. Moreover, real-world ICSs are dynamic and operate in noisy environments: these factors may hamper the correct functioning of the detection system because they can "shift" the normal behaviour. In such scenarios, the main challenges are to $(i)$ timely update the model of normality upon changes in the production workflows, and ( $i$ i) periodically refresh the detection thresholds. Previous works either do not tackle such challenges [3], [4] or require highly-specialised human intervention to update the parameters of the model [5], [6].

This paper tackles the aforementioned challenges by proposing DAICS (Deep learning Anomaly detection in Industrial Control Systems), an anomaly detection solution for ICSs based on the one-class classification paradigm. DAICS combines a database-oriented management approach and a deep learning architecture to model the normal behaviour of the ICS. The proposed neural network relies on wide and deep learning and convolutional layers to memorise and generalise the characteristics of the ICS. Wide and deep learning is a technique that has been recently proposed to improve the performance of recommender systems [7]. Convolutional layers, introduced by Yann LeCun in 1990 [8], apply small filters to detect patterns in the input while requiring a limited number of trainable parameters. Convolutional layers, and Convolutional Neural Networks in general, have become very popular in many application areas, including network security [9]-[11] and anomaly detection in ICSs [12]-[14].

As anticipated above, a common issue of one-class classification mechanisms is the high false alarm rate caused by the progressive evolution of the normal behaviour of the ICS with respect to the initial one, based on which the model was trained. This phenomena, called domain shift, can be caused by changes in the industrial workflow, degradation of devices and communication links over the time, installation/removal of devices, updates in the devices' firmware or configuration, or to external noise on the communication channels. DAICS implements an automatic threshold tuning algorithm and the 
so-called few-time-steps algorithm, the latter based on the fewshot learning paradigm [15], [16], to quickly update the model with the latest changes of the ICS normal behaviour.

We evaluate DAICS using datasets collected from the Secure Water Treatment (SWaT) and Water Distribution (WADI) testbeds, which are widely used for security research in ICSs [17]. The datasets comprise a training set containing only normal records, and a test set with normal and anomalous records. The anomalies in the test sets are real-world attacks targeting the integrity and the availability of the testbeds [18]. DAICS has been compared with state-of-the-art solutions in terms of anomaly detection accuracy and robustness to Gaussian noise.

The contributions of this work can be summarised as follows:

- An Anomaly Detection System (ADS) that has a modular architecture to fit large ICSs. The key components of the proposed ADS is a 2-branch neural network, in which a wide branch is designed to memorise the existing relations between the input features, while a deep branch generalises the model to unknown relations.

- An automatic threshold tuning technique that dynamically tunes the detection threshold based on the prediction error observed on the live ICS.

- A sensitivity analysis of hyperparameters of the proposed ADS using two real-world datasets, namely, the SWaT and WADI. The analysis provides practical insights to improve the performance under different operating conditions.

DAICS extends our previous work called AADS (Adaptive Anomaly Detection in industrial control Systems) [19]. With respect to AADS, DAICS scales better to larger ICSs, adopts an automated threshold tuning mechanism based on deep learning, and it has been tested on two public datasets: SWaT (like AADS) and WADI. The comparison presented in this paper shows that DAICS improves AADS in terms of precision (fewer false positives) and robustness to additive noise on the SWaT dataset.

The rest of this paper is organised as follows: Section II reviews state-of-the-art works on anomaly detection in ICSs. Section III defines the threat model used in this research. We describe the SWaT and WADI datasets in Section IV The problem statement is provided in section $\mathrm{V}$. Section VI introduces the proposed anomaly detection framework. Experimental setup and the evaluation results are presented in section VII. We conclude this paper in section VIII

\section{RELATED WORK}

This section reviews recent research studies on anomaly detection in ICSs. Particular attention is given to those works focusing on water treatment plants and validated using the SWaT and WADI datasets, as their performance is discussed in Section VII for state-of-the-art comparison.

In [5], Goh et al. present an approach based on a Recurrent Neural Network (RNN) and the Cumulative Sum (CuSum) technique to detect the anomalies. CuSum sets upper and lower control limits for the prediction error in each sensor and actuator. An anomaly is detected when the prediction error is outside such limits. Besides suffering from a high false-positive rate, the main limitation of this approach is that it might require an expert human intervention to tune and update the CuSum limits.

Kravchik et al. [20] propose a solution for detecting anomalies using convolutional and recurrent neural networks. The key aspect of this work is a statistical approach for anomaly detection based a normalised value of the prediction error. The normalisation is computed by using the mean and standard deviation of the prediction error of the benign samples recorded during normal operations of the water treatment plant, as recorded in the SWaT dataset. However, the authors do not explain how such statistical properties are updated in the case of changes in the production environment, quite frequent in realworld environments, as discussed in Section [1] An extension of this work can be found at [4], in which the authors present their preliminary results (the paper has not been peer-reviewed at the time of writing) on SWaT, WADI and BATADAL datasets. In addition to the 1D-Convolutional Neural Network (CNN) proposed in the first paper, the authors explore the performance of two more models such as Undercomplete Auto-encoder (UAE) and windowed-Principle Component Analysis (PCA). We compare the performance of DAICS and these works in Section VII

The approach presented in [21] is based on computing the distance between current and previous devices states. It is assumed that the distance should stay below a threshold during normal operations. The proposed solution has been tested only on the SWaT dataset, where it shows similar performance to DAICS in terms of F1 score, as discussed in Section VII-C However, the number of detected attacks is not shown in the paper, hence preventing a full understanding of the effectiveness of the approach.

The anomaly detection solution proposed in $[6]$ is based on an Multilayer Perceptron (MLP) and relies on a threshold applied to a weighted sum of the prediction errors of all sensors and actuators. Low weights are assigned to those devices whose normal behaviours are hard to predict. This strategy, adopted to increase the performance on the SWaT dataset, shows a high rate of false negatives, which leads to a low number of detected attacks, as shown in Section VII.

TABOR [3] is an anomaly detection solution validated on the SWaT dataset. TABOR combines two different models, namely: Probabilistic Deterministic Finite Automaton (PDFA), and a Bayesian Network (BN). The final anomaly detection is based on a combination of results from the two models. Also in this case, the authors do not address the problem of updating the model in case of changes in the normal operations of the ICS. Updating the model here appears significantly cumbersome due to the complex characterisation of the interaction between sensors and actuators needed to build the model.

In general, ADSs rely on a threshold that acts as the maximum allowed prediction error during normal operations, above which a systems state is considered anomalous. The threshold is either selected empirically by an expert [20] or based on the prediction error on the training set [6], [21]. In this paper, we propose a technique for automated threshold tuning, which dynamically adjusts the threshold on test time, improving the ability of DAICS to adapt to the normal 
behaviour evolution. In [22], [23], Markov chains and support vector regression are employed to dynamically tune thresholds for anomaly detection in network traffic (e.g., port scans, brute force attacks, SQL injections, etc.) and robot-assisted feeding. To the best of our knowledge, DAICS is the first anomaly detection solution for water treatment plants (and ICSs in general) to implement an automated threshold tuning mechanism.

In summary, a common drawback of available solutions is that they are not flexible enough to quickly and efficiently adapt to changes in the production environment. In a water treatment plant, examples of such changes are: increasing the size of a water tank or replacing a motorised valve with another with different operation modes. Instead, our approach is based on a novel algorithm called few-time-steps, presented in Section VI-E, that fine-tunes the neural network according to the changes in the normal behaviour of the ICS. The proposed algorithm uses a small amount of data to update the weights of the neural network and requires minimal human intervention. This is supported by an automated threshold tuning technique, which adds to DAICS ability to cope with the dynamic behaviour of industrial environments. Moreover, the proposed neural network architecture is designed to be scalable, so it can model the normal behaviour of large ICSs.

\section{THREAT MODEL}

We assume that attacker's capabilities include gaining remote access to the networked control system, to the Supervisory Control and Data Acquisition (SCADA) workstation, or can physically compromise sensors and actuators within the ICS. We also assume that the attacker has domain knowledge of the targeted ICS, e.g., the physical property measured by each sensor and the physical consequences of actuation commands. The attacker's goal is to use the above capabilities and knowledge to damage or modify the ICS operations. This includes: (i) altering the state of actuators through a MITMlike attack, in which the actuator receives commands from the attacker instead of a PLC, (ii) sending spoofed sensors readings to the PLC to drive the PLC to take wrong decisions, and (iii) tampering with the PLC firmware aimed to take the ICS out of service or to change the programmed logic (e.g., the Stuxnet worm [2]).

\section{DATASETS}

We evaluate DAICS using two popular public real-world datasets, namely Secure Water Treatment (SWaT) and Water Distribution (WADI). In this section we provide a short introduction to the main properties of the two dataset, also summarised in Table I.

TABLE I: Properties of the SWaT and WADI datasets.

\begin{tabular}{lccccc}
\hline Dataset & Records & $\begin{array}{c}\text { Duration } \\
\text { (days) }\end{array}$ & Attacks & Sensors & Actuators \\
\hline SWaT & 946,722 & 11 & 36 & 25 & 26 \\
WADI & $1,209,610$ & 16 & 15 & 69 & 54 \\
\hline
\end{tabular}

\section{A. The SWaT dataset}

This dataset has been collected from the SWaT testbed, a reduced version of an operational clean water treatment plant [17], [24]. The water treatment process is monitored by a SCADA workstation and is divided into six sub-processes, including raw water supply and storage, ultra-filtration and backwash. The sub-processes are controlled by a pair of PLCs that communicate with sensors (water flow indication transmitters, level indicator transmitters, analyser indicator transmitters, such as $\mathrm{pH}$ analysers, and ultra-violet modules) and actuators (e.g., pumps, motorised valves). The PLCs collect the readings from the sensors that monitor the status of the physical process, and send actuation commands to the actuators. Based on the PLC's internal logic, such commands may be used to either change or keep the current state of an actuator.

The dataset consists of 946,722 records of sensors and actuators collected during 11 days of operation at a rate of one sample per second. Each record contains 51 attributes, representing 25 sensors readings and 26 actuators states. The dataset is divided into a 7-day portion of normal operations, which has been used as the training set, plus a 4-day portion of normal activity combined with 36 attacks generated by following the attack model in [18] (the test set). $20 \%$ of the training set is used for validation (validation set).

The attacks in the test set are of duration ranging between two minutes and nine hours, and involve one or multiple devices at the same time. Attacks include spoofing the readings of sensors or reversing the operation states of actuators. For instance, in the first attack, the state of a motorised valve is switched from closed to open for 15 minutes aiming to cause a tank overflow. In another attack, the value reported by a water level sensor is decreased by $0.5 \mathrm{~mm} / \mathrm{sec}$ for seven minutes, again with the intention of causing a tank overflow. A more detailed description of these attacks is available in [24], [25].

\section{B. The WADI dataset}

The WADI testbed is a reduced version of a real-world water distribution network [17], [26]. The testbed network consists of three sub-processes, or stages, including a primary grid for water supply, a secondary grid for water distribution to six consumer tanks, and a return-water grid that handles the excess of water from the consumer tanks. Similarly to the SWaT testbed, also WADI is supervised by a SCADA workstation. Moreover, each sub-process is controlled by a PLC communicating with sensors and actuators. Sensors include water flow indication transmitters, level indicator transmitters, analyser indicator transmitters and pressure meters. Like the SWaT testbed, actuators include pumps and motorised valves.

The WADI dataset comprises 1,209,610 records, each with 123 attributes divided into 69 sensors readings and 54 actuators states collected during a period of 16 days. Normal operation conditions have been recorded during the first 14 days, split into training (95\%) and validation (5\%) sets. The last two days, with normal activity and 15 attacks, are used as test set. The attacks follow the same model used in the SWaT dataset and described in [18], although with shorter duration (between 2 and 30 minutes). For instance, one attack cuts off the water 
supply to consumer tanks; another one aims at increasing the level of chemicals in the water by turning off a sensor and sending false readings to the PLC for ten minutes. More details and examples on the WADI attacks can be found in [26].

\section{Problem Statement}

Unsupervised anomaly detection solutions for ICSs are usually built using the so-called one-class classification technique. The basic idea is to build a model of the normal behaviour of the industrial process and to consider as anomalous every event that does not fit the model. The main challenge with such approaches is dealing with the domain shift, which originates from a gap in the data distribution between the training and unseen data [27], [28]. The tangible effect of the domain shift is an increase of false alarms generated by the anomaly detection system due to normal events classified as anomalies.

The domain shift problem is present in the SWaT dataset, where we can observe changes in the normal behaviour of some devices across the training and test sets. For instance, during the normal operation, the pump P102 has a single state of value 1 in the training set, then it takes an additional "normal" state of value 2 in the test set. Also, the probability distribution of some sensors changes between the two sets. For example, in the training set the output of the Analyser Indication Transmitter AIT201 ranges in interval $[251,272] \mu S / \mathrm{cm}$ (micro Siemens per centimetre), while in the test set it ranges in [168, 267] $\mu S / \mathrm{cm}$ with a substantial different distribution, as illustrated in Figure 1. Another non-trivial and representative example is the presence of redundant devices, such as the redundant pump P102 in the SWaT testbed. A redundant pump is always off until the primary pump stops working unexpectedly. In this case, the PLC turns on the redundant pump instantaneously to take over the work of the primary pump. If such a process is not covered in the training set, the forecasting model will consider the operations of the redundant pump as anomalies.

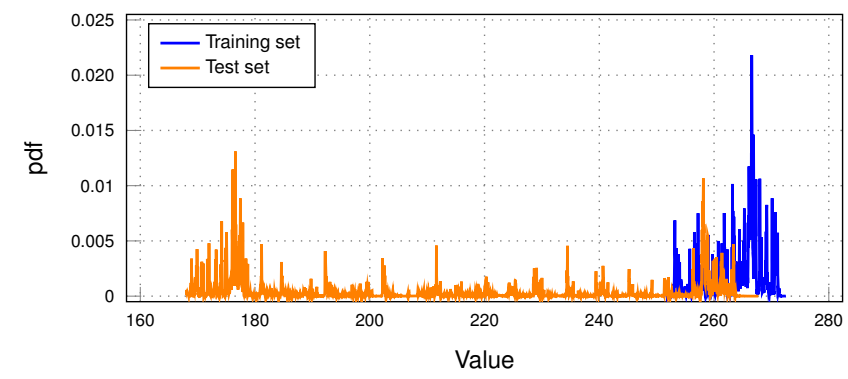

Figure 1: The probability density function for sensor AIT201. It shows the variation in the normal behaviour between training and test sets.

The domain shift problem can be also observed in the WADI dataset. For instance, the records of one of the Flow Indication Transmitters range within $[0,2.3] \mathrm{m}^{3} /$ hour of water in the training set, while the range changes to $[0,3.3] \mathrm{m}^{3} /$ hour in the test set.

The characterisation of the normal behavioural evolvement is still a challenge for the implementation of the anomaly detection solutions in real-world systems [29], [30]. Current
TABLE II: Glossary of symbols.

\begin{tabular}{|l|l|}
\hline WDNN & Wide and Deep Neural Network \\
\hline TTNN & Threshold Tuning Neural Network \\
\hline$W_{\text {in }}$ & Input time window \\
\hline$W_{\text {out }}$ & Output time window \\
\hline$W_{a n o m}$ & Anomaly waiting time \\
\hline$H$ & Horizon \\
\hline$G$ & Number of output section of WDNN \\
\hline$\mu_{o u t}[g]$ & Output section $g$ of WDNN \\
\hline$m_{a c}$ & Total number of actuators \\
\hline$m_{s e}$ & Total number of sensors \\
\hline$m_{s e}^{g}$ & Number of sensors of output section $g$ \\
\hline $\mathrm{MSE}_{g, t}$ & Prediction error on section $g$ at time $t$ \\
\hline $\mathrm{MSE}_{g,[a, b)}$ & List of prediction errors on section $g$ between time $a$ and $b-1$ \\
\hline$T_{g}$ & Anomaly threshold for section $g$ \\
\hline$\nu_{t}$ & Actuator states recorded at time $t$ \\
\hline
\end{tabular}

research in ICSs have tackled the domain shift problem by only focusing on adjusting the parameters of the detection algorithms or excluding devices with domain shift from the modelling process, as explained before in Section III. However, we argue that tuning the detection parameters without updating the model of normality is not sufficient to cope with dynamic environments such as ICSs.

In the next section, we present our solution for anomaly detection in ICSs that encompasses a lightweight technique, called few-time-steps learning, which is inspired by the fewshot learning paradigm [16], [31]. The few-time-steps learning technique updates the initial model of the industrial process throughout its evolvement in the hardware and software configuration, hence minimising the number of false positives caused by the domain shift phenomena.

\section{THE DAICS FRAMEWORK}

DAICS defines a model of the normal behaviour of the ICS combining a neural network with a database-like approach. Deep learning techniques are used to characterise the continuous readings of sensors, whose values are usually sampled by a PLC very frequently, while the database memorises the states of actuators, as commanded by the PLC. DAICS includes the few-time-steps learning algorithm to update the model based on the normal behavioural evolvement of the ICS. The detection of anomalies is determined with a threshold that is automatically adjusted using the prediction error.

\section{A. Prediction of sensor states}

The normal behaviour of sensors is modelled using a deep learning architecture called Wide and Deep Neural Network (WDNN). The architecture of WDNN, shown in Fig. 2 is inspired by the recommender system introduced in [7], where the authors trained a wide and deep neural network to recommend apps based on the user's query and preferences. Our neural network has two goals: memorization through the wide branch and generalization through the deep branch. Memorization means learning the relationship between featurepairs in the training set, hence recording the co-occurrence of combinations of sensors values. Generalization means the ability to explore relationships that do not exist in the training set. 


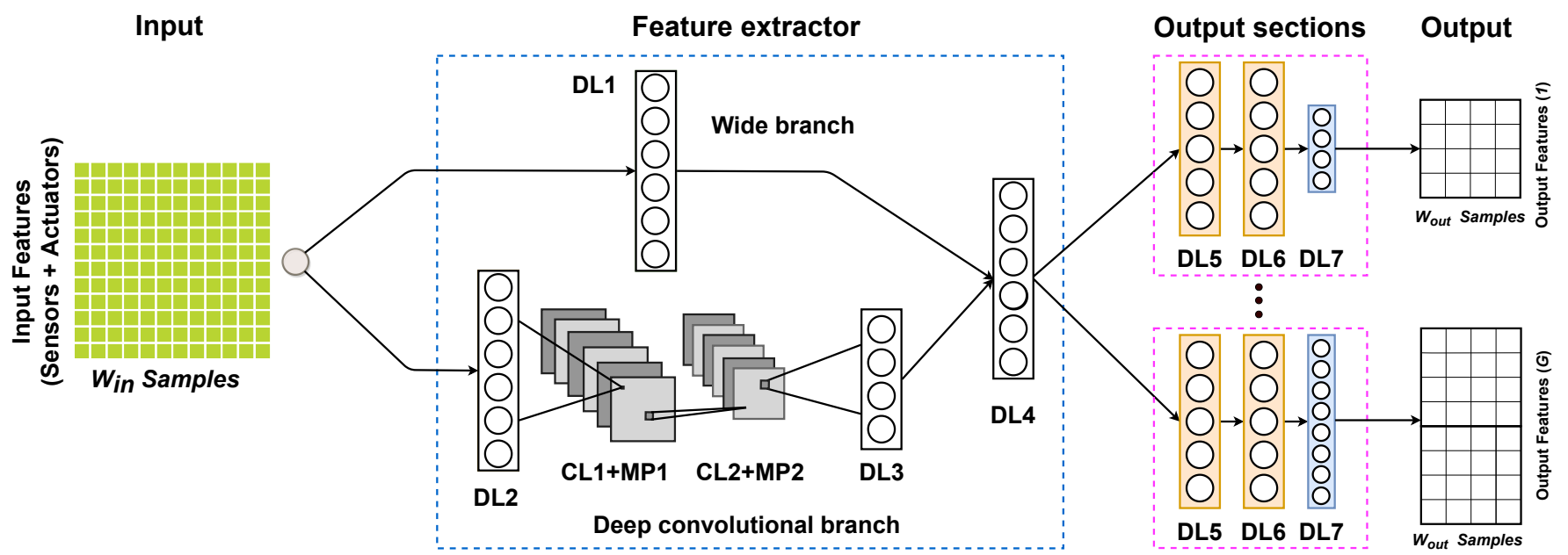

Figure 2: Architecture of WDNN. The prediction of the sensors states,i.e. the output features, is computed combining the information from both sensors and actuators. Note that the size of DL7 varies according to the number of output features in each output section.

Like in our previous work [19], the neural network comprises a Feature extractor section that learns the relations between all sensors and actuators during the normal operation of an ICS and extracts the features required to predict the normal states of sensors. Instead, the output section is split into multiple branches to serve large-scale ICSs where the sensors are controlled by several PLCs, usually specialised on a specific part of the industrial process with specific behaviour and anomaly threshold.

Architecture. The neural network takes as input an array $X$ of data samples collected during a time window of length $W_{i n}$ seconds, corresponding to $W_{i n}$ samples, as the dataset was collected at a sampling rate of one sample per second. The size of $X$ is $m \times W_{i n}$, where $m=m_{s e}+m_{a c}$ is the number of features for each sample including the state of $m_{s e}$ sensors and $m_{a c}$ actuators taken at time $t$. Please note that we use the features from both sensors and actuators because the behaviour of the sensors depends on their current states and the actions taken by actuators. The output $Y$ is the predicted readings from sensors during a future time window $W_{\text {out }}$. The two timewindows $W_{\text {in }}$ and $W_{\text {out }}$ are separated by a time interval called horizon $H$. This separation prevents the forecasting model from copying the last values of the input time window $W_{i n}$ into $W_{\text {out }}$, as pointed out by the authors of [6].

As shown in Fig. 2, the neural network architecture comprises two convolutional layers and seven fully connected layers. The fully connected layer DL1 defines the so-called wide branch used for the memorisation of the normal state of sensors and actuators using cross products between the input features.

The deep branch provides the level of generalisation necessary for correctly handling the events not covered in the training set, aiming to minimise the prediction error in the case of new input states. The deep branch is formed with a sequence of one fully connected layer (DL2), two onedimensional convolutional layers (CL1 and CL2) each one followed by a max-pooling layer (MP1 and MP2), and finally an additional fully connected layer (DL3). Layer DL2 transforms the input size by increasing the size by a factor of three, acting as a feature enrichment technique. As shown in other works (e.g., [20]), one-dimensional CNNs are particularly suited for modelling time series data. The purpose of layers CL1 and CL2 is to model the data collected from sensors and actuators in a specific time window. For max pooling, we down-sample the output of each convolutional layer by a factor of 2 . The final fully connected layer DL3 re-shapes the output of the deep branch to allow its concatenation with the output of the wide branch.

Both branches are aggregated in another fully connected layer (DL4) followed by multiple dense output sections, each one consisting of the three fully connected layers DL5, DL6 and DL7. Each output section learns the most relevant information from the aggregation layer in order to predict the normal behaviour of a group of sensors controlled by a specific PLC.

Each fully connected layer can be described as $Y=$ LeakyReLU $\left(\mathbf{W}^{T} X+\mathbf{b}\right)$, where $Y$ is the output, $X$ input, $\mathbf{W}$ is an array of weights the model learns during the training, and $\mathbf{b}$ is the bias. We introduce non-linearity in the model by using the leaky rectified linear activation function defined as follows: $\operatorname{LeakyReLU}(x)=\max (0, x)+0.01 * \min (0, x)$. Similarly, the convolutional layers can be described as $Y_{i}=$ $\operatorname{LeakyReLU}\left(\operatorname{Conv}\left(X, \mathbf{W}_{i}, \mathbf{b}_{i}\right)\right)$, where $Y_{i}$ is the output of the convolution on the input $X$ using the $i$-th filter with weights $\mathbf{W}_{i}$ and bias $\mathbf{b}_{i}$.

Cost function. The neural network presented above has been trained to minimise the Mean Square Error (MSE) cost function by iteratively updating all the weights and biases contained within the feature extractor and all output sections. The cost function computes the error between the prediction of the model and the corresponding observed sensor values. Hence, minimising the cost, we reduce the prediction error. At the training stage, the cost function for a batch size of $s$ samples (i.e., $s$ different time windows) in a specific output 
section $g$ can be formally written as:

$$
c_{g}=\frac{1}{s} \sum_{t=1}^{s}\left(\frac{1}{m_{s e}^{g}} \sum_{i=1}^{m_{s e}^{g}}\left(Y_{t}[i]-\tilde{Y}_{t}[i]\right)^{2}\right)
$$

where $Y_{t}[i]$ is the predicted value for sensor $i$ at sample $t$ (i.e. time-step $t$ ), while $\tilde{Y}_{t}[i]$ is the corresponding observed sensor value (the value present in the training set). $m_{s e}^{g}$ is the number of sensors in an output section $g$. The final cost $c$ is the sum of costs of all $G$ output sections $c=\sum_{g=1}^{G} c_{g}$. This cost is minimised by using the optimisation algorithm Stochastic Gradient Descend (SGD) [32].

\section{B. Anomaly detection in actuators}

Actuators in the SWaT and WADI testbeds include pumps and motorised valves. The pumps are arranged in pairs of primary and redundant hot-standby pumps. A redundant pump is turned on only in the case the respective primary pump stops working. This operational mode complicates building a forecasting model that predicts the actuator states, as some actuators (such as the redundant pumps), are rarely used during the normal operations. As a consequence, after measuring a high prediction error with Deep Learning (DL)-based methods due to lack of normal operation records, we designed a light and straightforward approach based on querying a database containing all the normal actuator states. The database entries are $n$-tuples, where $n$ is the number of actuators in the testbed ( $n=26$ in the SWaT, $n=54$ in the WADI). Each entry is a combination of actuators states labelled as normal, for a total of 146 entries available in the SWaT dataset and 2001 entries in the WADI dataset. An example of tuple from the SWaT testbed is provided in Table III

TABLE III: Tuple in the database of actuators normal states.

\begin{tabular}{l|ccccccc}
\hline Actuator & MV101 & P101 & P102 & $\ldots$ & P601 & P602 & P603 \\
\hline Tuple & 2 & 2 & 1 & $\ldots$ & 1 & 1 & 1 \\
\hline
\end{tabular}

At testing time, the combinations in the test set with no occurrences in the training set are marked as anomalies, as explained in Section VI-C.

\section{Detection logic}

DAICS operates on batches of actuators and sensors values retrieved from the PLCs at regular time intervals of duration $s$ seconds. For the sake of simplicity, we assume that $s$ also corresponds to the number of readings in one batch for each device (like in the two datasets used in the experiments). We therefore define $I=[\bar{t}, \bar{t}+s)$ the time interval under analysis (where $[a, b)$ indicates the interval between $a$ and $b-1$ ). Given the observation time $t \in I$, DAICS verifies whether the current combination of actuator values is present in the database $A$ built at the training stage. An anomaly is reported otherwise.

In the case of the sensors, the values observed at time $t$ are compared against those predicted by WDNN using past values of sensors and actuators, as previously explained in Section VI-A. More precisely, the values observed at time $t \in I$ are compared with the first element of the output of

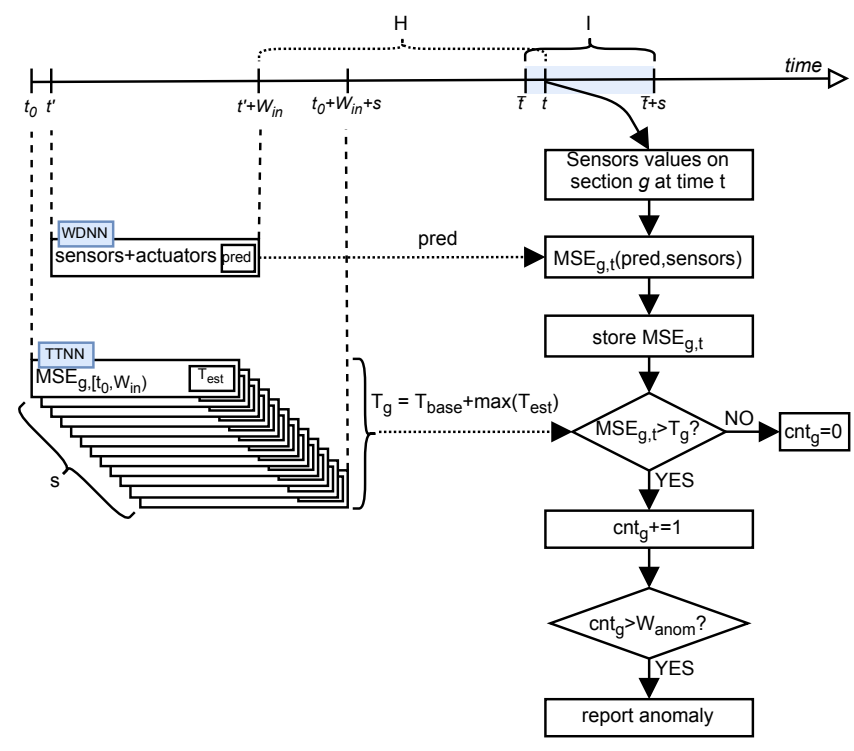

Figure 3: Detection process on section $g$ of sensors through WDNN and adaptive thresholds.

WDNN obtained from values of sensors and actuators collected in the time window $\left[t^{\prime}, t^{\prime}+W_{i n}\right.$ ) (one input sample for WDNN), where $t^{\prime}=t-H-W_{i n}$. The MSE value of each WDNN output section is evaluated against an adaptive threshold to determine the presence of any anomalies.

The detection process on one single output section $g$ of the neural network is summarised in Figure 3, which also depicts an adaptive threshold mechanism for automatically tuning the anomaly threshold based on observed noise conditions on the sensors readings. Such a mechanism is described in Section VI-D. The region of the timeline highlighted in light blue represents the time interval $I$ described above.

More precisely, the samples observed on the group $g$ of sensors at time $t$ are identified as anomalous when the anomaly condition $\mathrm{MSE}_{g, t}>T_{g}$ is met, where $T_{g}$ is a threshold on the prediction error for the group of sensors $g$. Using the same notations as for the cost function in Equation 1, we define $\mathrm{MSE}_{g, t}$ as follows:

$$
\operatorname{MSE}_{g, t}=\frac{1}{m_{s e}^{g}} \sum_{i=1}^{m_{s e}^{g}}\left(Y_{t}[i]-\tilde{Y}_{t}[i]\right)^{2}
$$

To reduce the false positives caused by sudden changes in the underlying physical process (as also reported in [20]), DAICS only reports an anomaly at time $t$ on the sensors if the anomaly condition has been also previously observed for $W_{\text {anom }}$ consecutive sampling intervals. In Figure 3 , this process is represented through increasing the counter $c n t_{g}$.

In summary, the anomaly condition can be expressed as in the following Equation 3 .

$$
L_{t}= \begin{cases}1, & \text { if } \exists g \in[1, G] \text { s.t. } \\ & \operatorname{MSE}_{g, i}>T_{g} \forall i \in\left[t-W_{\text {anom }}, t\right] \\ 1, & \text { if } \nu_{t} \notin A \\ 0, & \text { otherwise }\end{cases}
$$


where $\nu_{t}$ is the combination of the actuators values observed at time $t$, while $L_{t}=1$ defines the anomaly condition at time t. $W_{\text {anom }}$ is one of the hyper-parameters used to tune DAICS, as reported in Section VII

\section{Threshold Tuning}

The industrial environments are usually subject to various sources of noise. In particular, the electromagnetic noise can interfere with the communication within the ICS, hence compromising the operations of anomaly detection systems [33], [34]. The main challenge is finding the correct threshold needed to classify an event either as anomaly or as normal activity. Previous works tackled this problem empirically, by tuning the threshold at test time as a hyper-parameter [5], [6], [20]. While this technique produces good results in the laboratory when using static datasets such as SWaT and WADI, online systems can hardly afford long threshold tuning sessions for updating the thresholds upon new noise levels. Instead, here we propose an adaptive technique that dynamically tunes the threshold based on the prediction error observed previously.

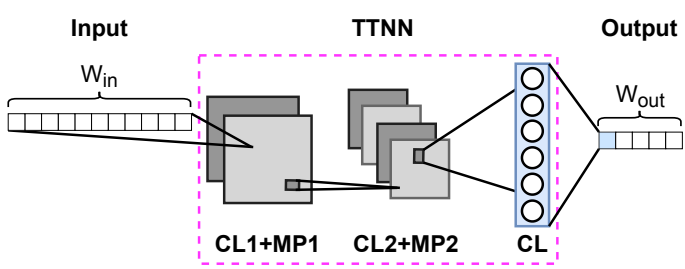

Figure 4: Architecture of TTNN. Only the first element of the output is kept. The size of the 1-dim kernel of CL1 and CL2 is 2 with stride 1 .

Ali et al. [22] used a machine learning technique to estimate the threshold based on the historical prediction error of their ADS. In this paper, we treat the prediction error MSE as an univariate time series that is modelled using a neural network that we called TTNN, whose architecture is depicted in Figure 4 . We use $G$ instances of TTNN, one for each output section of the WDNN model, to tune the thresholds $T_{g}, g \in[1, G]$. Each instance is trained with the prediction error $\mathrm{MSE}_{g}$ measured on a single output section $g$ on the validation set, which only contains benign records, as the training set. The trained model is used in the online system to compute the optimal anomaly threshold $T_{g}$ using past prediction errors. As represented in Figure 3, the threshold $T_{g}$ used for anomaly detection on sensors values collected in time interval $[\bar{t}, \bar{t}+s)$ is obtained using past prediction errors computed in time interval $\left[t_{0}, t_{0}+\right.$ $\left.s+W_{i n}\right)$, where $t_{0}=\bar{t}-H-W_{i n}$.

As shown in Figure 4, TTNN consists of two 1-dimensional convolutional layers, each followed by a max pooling layer, and of one final fully connected classification layer, represented in the figure as CL1, MP1, CL2, MP2 and DL respectively. The input is a time series of prediction errors measured for the samples collected in past time window of length $W_{\text {in }}$ seconds, the output contributes to the estimation of the optimal anomaly threshold for the current sensors states. A detailed description of the threshold tuning process is described in Algorithm 1 .

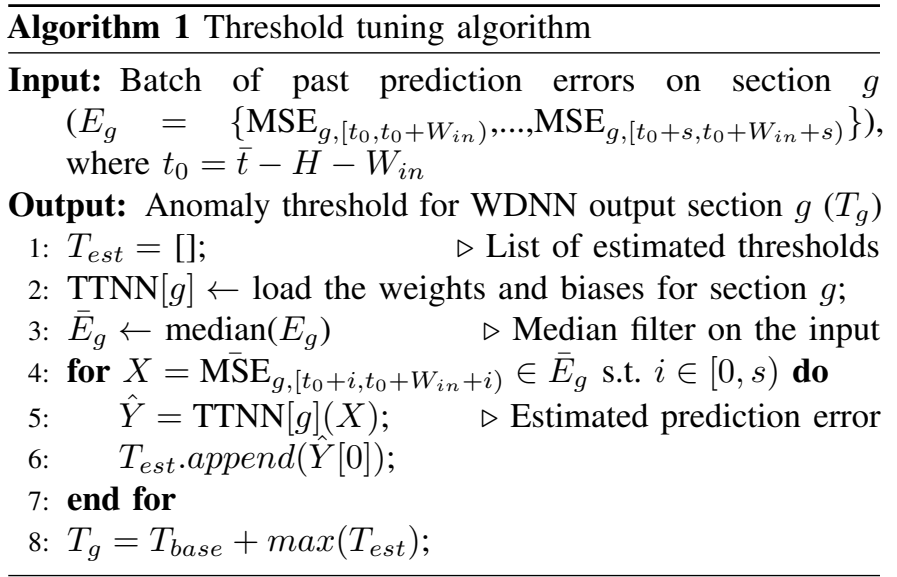

Referring to the timeline depicted in Figure 3 , the anomaly threshold used at time $t \in[\bar{t}, \bar{t}+s)$ for output section $g$ is computed by using a batch $E_{g}$ of $s$ samples defined as time series of past prediction errors of length $W_{i n}$, starting from $\mathrm{MSE}_{g,\left[t_{0}, t_{0}+W_{i n}\right)}, \mathrm{MSE}_{g,\left[t_{0}+1, t_{0}+W_{i n}+1\right)}$, to $\mathrm{MSE}_{g,\left[t_{0}+s, t_{0}+W_{i n}+s\right)}$.

At line 3 in of the algorithm, a median filter is applied to the input batch of past prediction errors $E_{g}$ to reduce the impact of short-term changes of the prediction error on the threshold tuning process. Median filtering is accomplished by sliding a window over $E_{g}$ while computing the median value of the elements of $E_{g}$ under the window. The resulting median values are stored in array $\bar{E}_{g}$. One variable to consider in this process is the size of the median filter. In this regard, we experimented with values between 50 and 120 . As reported in Table IV we obtained the best results with median filter size of 59 . In the loop at lines 4-7, the algorithm computes the estimated prediction error using the median values in $\bar{E}_{g}$. As shown at line 6 , only the first element of the output layer is memorised for further processing.

The threshold $T_{g}$ is computed at line 8 as the maximum of the predicted thresholds plus the offset $T_{\text {base }} . T_{\text {base }}$ is set as the sum of the mean plus the standard deviation of the prediction error on the validation set, and is kept constant after the deployment. This offset is added to reduce the sensitivity of the WDNN to small variations on sensors values, hence reducing the false positives.

As a final step, the algorithm executes one SGD epoch to update weights and biases of each of the $G$ instances of TTNN using the respective input batch $E_{g}$.

\section{E. The few-time-steps algorithm}

The few-time-steps algorithm has been designed to efficiently reconfigure DAICS in a production environment in the case an anomaly is identified by the system and then recognised as false alarm by the technician.

In such a situation, DAICS is triggered by the technician to update the database $A$ of actuators states and to tune the output section of the pre-trained WDNN. The only assumption is that the technician can recognise false alarms caused by changes in the normal operating condition of the ICS (e.g., changes 
in the hardware/software configurations). Unlike state-of-theart solutions, the proposed approach only assumes a domainspecific understanding of the ICS operations, without requiring a deep knowledge of the algorithms and their thresholds and hyper-parameters.

Of course, the number of human interventions needed to handle the false alarms is an important parameter to determine the usability of the anomaly detection system. If the system is too sensitive, the technician would be overwhelmed by a large number of alarms to be verified, making it hard to spot those that are due to normal changes in the ICS behaviour, hence false. On the contrary, a conservative approach might not reveal true malicious activities or anomalies. Both cases can render the system ineffective, if not unusable. The sensitivity of DAICS to this parameter will be analysed in Section VII.

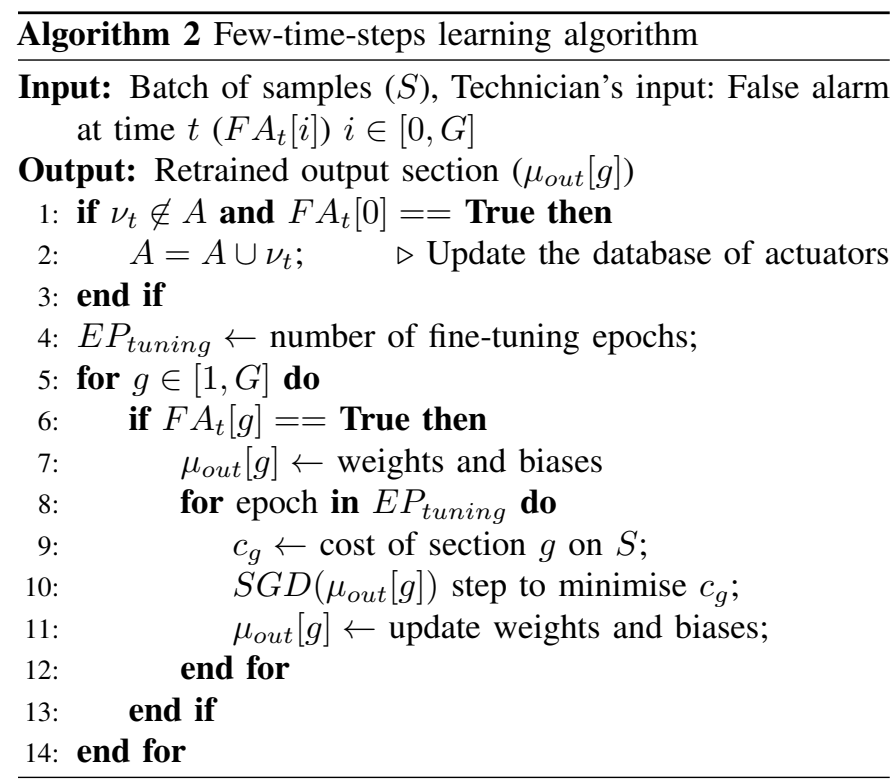

Algorithm 2 is called upon an technician's decision that an alarm detected by the system at time $t$ is false. This means that Algorithm 2 is called when both of the following conditions are satisfied: the anomaly condition $L_{t}=1$ and at least one of the elements of the boolean vector $F A_{t}[i](i \in[0, G])$ is True. $F A_{t}$ indicates where the false alarm has been detected either among the actuators $\left(F A_{t}[0]=\right.$ True $)$, or in one or more groups of sensors $\left(F A_{t}[i]=\right.$ True, $\left.i \in[1, G]\right)$, or both

In the first case, with $F A_{t}[0]=$ True, the algorithm adds the combination of actuator states $\nu_{t}$ to database $A$ (lines 1-3), as the technician has determined that $\nu_{t}$ is normal and must be treated as such by the system. In lines 5-14, the output sections of the neural network that have produced the false alarm are updated. Specifically, in lines 8-12, SGD is employed to finetune the output section through multiple gradient steps. We calculate the prediction loss for the data samples aggregated in a batch of $S$ samples containing the false alarm ( $S$ is passed as an input to algorithm). The optimiser minimises this loss by tuning the parameters of the output layers DL, DL6, and DL7. After $E P_{\text {tuning }}$ optimisation steps (around $400 \mathrm{~ms}$ on average for 100 epochs on our testing environment described in Section VII-A, the updated output section $\mu_{\text {out }}[g]$ replaces the previous one in the anomaly detection process (line 11).
Note that, grouping the sensors into different sections speeds up the execution of the few time steps algorithm, since only a portion of the output section is updated in the case of false alarms.

\section{EXPERIMENTAL EVALUATION}

In this section, we present a detailed evaluation of DAICS obtained using the SWaT and WADI datasets presented in Section IV The evaluation comprises a comparison with stateof-the-art solutions in terms of detection accuracy, number of detected attacks and robustness to noisy data.

\section{A. Experimental Setup}

DAICS has been implemented in PyTorch 1.0 [35] and validated using a Singularity container [36] running in a shared machine configured with $16 \mathrm{CPU}$ cores, 64 GB virtual RAM and an NVIDIA 1080Ti GPU. The database of actuators normal states $A$ is implemented as a NumPy array populated using the training datasets. The maximum size of the array is less than $1 \mathrm{MB}$ for each of the two datasets. Prior to our experiments, we also normalised the sensor readings between 0 and 1 .

\section{B. Methodology}

As per convention in the literature, we configure and evaluate DAICS using the following metrics:

$$
\operatorname{Pr}=\frac{T P}{T P+F P} \quad R e=\frac{T P}{T P+F N} \quad F 1=2 \cdot \frac{P r \cdot R e}{P r+R e}
$$

where $P r=$ Precision, $R e=$ Recall, F1=F1 Score, TP=True Positives, $F P=$ False Positives, $F N=$ False Negatives. It is important to highlight that we adopt a point-based approach to compute these metrics. Thus, precision, recall and F1 score are computed using the labelled records (points) of the datasets, which report whether a record (a combination of actuators states and sensors readings recorded at a given time) is normal or anomalous. Like most of related works in the literature, we adopt this approach to tune the hyperparameters of WDNN and TTNN models, to assess the overall performance of DAICS and for comparison with the state-of-the-art.

We used a grid search strategy to explore the set of hyperparameters using F1 score as the performance metric. The final sets of hyper-parameters that maximise the F1 score on the two datasets are reported in Table IV.

These hyper-parameters are kept constant throughout our experiments presented below in this section, except when we study the sensitivity of DAICS to a specific hyper-parameter.

In our experiments, we measure the sensitivity of DAICS to noise. It is worth recalling that industrial control systems operate in hostile environments [37], where the communication channels are often subject to interference (e.g., in the case of employing wireless communication devices [38]). For evaluation purposes we add Gaussian noise to sensor readings of the SWaT dataset with mean $\mu=0$ and standard deviation $\sigma \in\{1,2,3,5,10,15\}$. The noise distribution and the values of $\mu$ and $\sigma$ have been selected based on similar assumptions made in other works regarding the noise in communication channels of networked control systems [33], [34]. 
TABLE IV: Hyperparameters.

\begin{tabular}{|c|c|c|}
\hline Hyperparameter & SWaT & WADI \\
\hline Horizon $(H)$ & 50 & 20 \\
\hline Input time window $\left(W_{i n}\right)$ & 60 & 50 \\
\hline Output time window ( $\left.W_{\text {out }}\right)$ & 4 & 4 \\
\hline $\begin{array}{l}\text { Anomaly time window } \\
\left(W_{\text {anom }}\right)\end{array}$ & 30 & 30 \\
\hline \multicolumn{3}{|c|}{ WDNN } \\
\hline Hyperparameter & SWaT & WADI \\
\hline Learning rate $(\alpha)$ & 0.01 & 0.001 \\
\hline Batch size $(s)$ & 32 & 32 \\
\hline Tuning epochs $\left(E P_{\text {tuning }}\right)$ & 100 & 100 \\
\hline DL1 Neurons & $W_{\text {out }}$ & $W_{\text {out }}$ \\
\hline DL2 Neurons & $3 * W_{i n}$ & $3 *\left(m_{s e}+m_{a c}\right)$ \\
\hline DL3 Neurons & $W_{\text {out }}$ & $W_{\text {out }}$ \\
\hline DL4 Neurons & 80 & 80 \\
\hline DL5 Neurons & $2.25 * m_{s e}^{g}$ & $2.25^{*} m_{s e}^{g}$ \\
\hline DL6 Neurons & $1.5^{*} m_{s e}^{g}$ & $1.5^{*} m_{s e}^{g}$ \\
\hline DL7 Neurons & $m_{s e}^{g}$ & $m_{s e}^{g}$ \\
\hline CL1 Kernels, Kernel size & 64,2 & 64,5 \\
\hline MP1 Pooling size & $(64,2)$ & $(64,2)$ \\
\hline CL2 Kernels, Kernel size & 128,2 & 128,5 \\
\hline MP2 Pooling size & $(128,2)$ & $(128,2)$ \\
\hline \multicolumn{3}{|c|}{ TTNN } \\
\hline Hyperparameter & SWaT & WADI \\
\hline Learning rate $(\alpha)$ & 0.01 & 0.01 \\
\hline Batch size $(s)$ & 32 & 32 \\
\hline Tuning epochs $\left(E P_{\text {tuning }}\right)$ & 1 & 1 \\
\hline Median kernel size & 59 & 59 \\
\hline CL1 Kernels, Kernel size & 2,2 & 2,2 \\
\hline MP1 Pooling size & $(2,2)$ & $(2,2)$ \\
\hline CL2 Kernels, Kernel size & $W_{\text {out }}, 2$ & $W_{\text {out }}, 2$ \\
\hline MP2 Pooling size & $\left(W_{\text {out }}, 2\right)$ & $\left(W_{\text {out }}, 2\right)$ \\
\hline DL Neurons & 1 & 1 \\
\hline
\end{tabular}

In this research, we do not evaluate the robustness of DAICS against adversarial noise, i.e., the noise added to the communication channels by an attacker intending to perturb the detection accuracy. Indeed, the defence against adversarial attacks in ICSs is a complex problem, and its discussion is outside the scope of this work.

The validation presented below is divided into three different experiments. In Experiment 1 and 2, we compare the performance of DAICS with relevant works in the state-of-the-art in terms of precision, recall, F1 score, and the number of attacks correctly detected. Experiment 1 evaluates DAICS using the SWaT dataset, while experiment 2 uses the WADI dataset. In Experiment 3, we evaluate the robustness of DAICS to additive noise applied on the SWaT and WADI test sets. We also compare the results with the frameworks proposed in [19], [20] in case of the SWaT dataset.

\section{Experiment 1: Detection accuracy in the SWaT}

As shown in Table V] DAICS outperforms existing stateof-the-art detection on the SWaT test set with $88.9 \%$ F1 score, and correctly recognises 33 out of 36 attacks in the test set. It is worth noting that DAICS also detects a higher rate of attacks during their execution (97\% against $93 \%$ of [20]), hence allowing the operator to activate the adequate countermeasures more promptly.

Few-time-steps algorithm. We also evaluated the contribution of the few-time-steps algorithm to the detection accuracy
TABLE V: State-of-the-art comparison (SWaT dataset). In parenthesis, the number of attacks detected after their end.

\begin{tabular}{lcccc}
\hline Architecture & Precision & Recall & F1 & $\begin{array}{c}\text { Detected } \\
\text { attacks }\end{array}$ \\
\hline DAICS & 0.9185 & 0.8616 & 0.8892 & $33(1)$ \\
AADS [19] & 0.866 & 0.861 & 0.863 & $33(1)$ \\
MLP [6] & 0.967 & 0.696 & 0.812 & 25 \\
CNN [20] & 0.867 & 0.854 & 0.860 & $31(2)$ \\
TABOR [3] & 0.861 & 0.788 & 0.823 & 24 \\
Windowed-PCA [4] & 0.92 & 0.841 & 0.879 & - \\
Online-ADS [21] & 0.8638 & 0.9064 & 0.8846 & - \\
\hline
\end{tabular}

of DAICS. To this aim, we repeated the experiment disabling the algorithm. As the SWaT test set contains normal records that are not present in the training set, the model of normal behaviour built with the training set leads to several false positives and, more precisely, to a low F1-score measure of $77.7 \%$ and 1251 false alarms (compared to the 645 experienced with the algorithm enabled).

The update process is fast. Indeed, with our setup, the execution of one cycle of the few-time-steps learning algorithm, including the $E P_{\text {tuning }}=100$ epochs, with a batch of 32 samples takes $400 \mathrm{~ms}$ on average. In an online system, the algorithm can be triggered by the operator upon identifying a false alarm (e.g., a known unstable sensor or actuator). It is important to stress that this is the only requirement for the operator, unlike other solutions where an in-depth knowledge of the underlying algorithms is necessary to update thresholds or other parameters.

Hyper-parameters. The sensitivity of DAICS to the hyperparameter $W_{\text {anom }}$ is presented in Figure 5. We remind that $W_{\text {anom }}$ is defined in Equation 3 as the number of consecutive times the prediction error MSE is higher of the anomaly threshold on the same output section of WDNN.

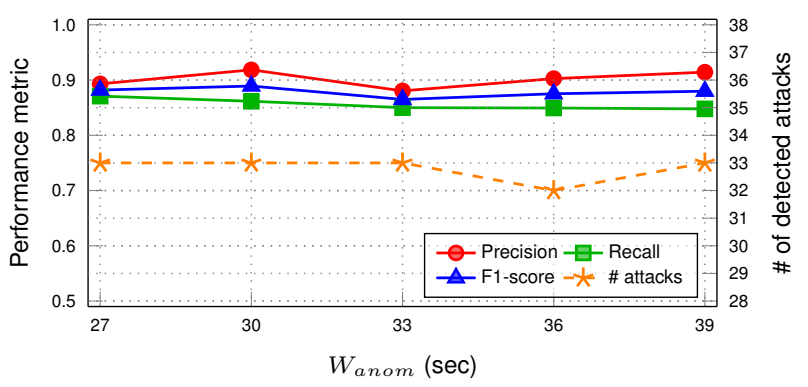

Figure 5: Sensitivity of WDNN to $W_{\text {anom }}$ on SWaT dataset.

Although the number of detected attacks is quite stable to 33 (except for $W_{\text {anom }}=36$ ), the highest F1 score (0.8892) is measured at $W_{\text {anom }}=30$.

False alarms. DAICS relies on the feedback from the technician to fine-tune the output sections with new information of the normal behaviour. Of course, the rate of technician's interventions is a relevant metric to consider, as too frequent false positives can undermine the usability of the system. With the chosen settings, we measured 645 false alarms in total on the test set of the SWaT dataset, equivalent to 6.6 human interventions/hour on average. Although this seems a 


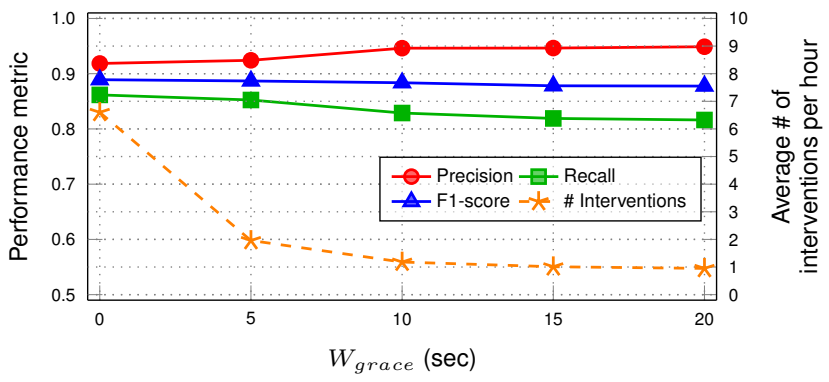

Figure 6: Sensitivity of WDNN to $W_{\text {grace }}$ on SWaT dataset.

manageable rate of interventions, in more complex industrial environments compared to the SWaT testbed.

One way to reduce the rate of intervention is to allow a grace time $W_{\text {grace }}$, through which alarms are reported to the technician only if they last for at least $W_{\text {grace }}$ seconds. Of course, this practice may hide short true positives, hence preventing the detection of a certain number of anomalies.

We tested the sensitivity of DAICS to the grace time by varying $W_{\text {grace }}$ from 1 to 20 seconds. In Figure 6 we can observe a minimal decrease of the F1 score $(0.877$ with $W_{\text {grace }}=20$ ) and a consistent reduction of the technician intervention rates (from 6.6/hour with no grace time, to 1.96/hour with $W_{\text {grace }}=5$, to $0.95 /$ hour with $W_{\text {grace }}=20$ ). Moreover, we did not experience any variations in the number of detected anomalies with $W_{\text {grace }}=5$ (still 33), while with $W_{\text {grace }}=20$ this number decreases to 28 , as somehow expected.

\section{Experiment 2: Detection accuracy in the WADI}

DAICS matches the state of the art results in terms of number of attacks detected, 14 out of 15 attacks in the WADI dataset, while it outperforms the other solutions in terms of F1 score, as summarised in Table VI.

TABLE VI: State-of-the-art comparison (WADI dataset).

\begin{tabular}{lcccc}
\hline Architecture & Precision & Recall & F1 & $\begin{array}{c}\text { Detected } \\
\text { attacks }\end{array}$ \\
\hline DAICS & 0.9083 & 0.7205 & 0.8036 & 14 \\
MAD-GAN [39] & 0.4144 & 0.3392 & 0.37 & - \\
1D CNN [4] & 0.697 & 0.731 & 0.714 & 14 \\
AE [4] & 0.834 & 0.681 & 0.750 & 14 \\
\hline
\end{tabular}

Few-time-steps algorithm. Given the setup described in section VII-A DAICS takes $800 \mathrm{~ms}$ to fine-tune an output section with 100 epochs and a batch of 32 samples. Like in the case of the SWaT dataset, we demonstrate how the few-timesteps algorithm contributes to keeping weights and biases of WDNN up-to-date with the changes of the normal behaviour. Indeed, when disabling the updating mechanism, the F1-score drops to 0.5621 , while the number of false alarms increases to from 226 to 684 .

Hyper-parameters. Increasing the anomaly window $W_{\text {anom }}$ determines an increase on the number of detected attacks, as shown in Figure 77. This is mainly due to the condition $\mathrm{MSE}_{g, i}>T_{g}$ in Equation 3, which yields to the detection of short attacks when $W_{\text {anom }}$ is large enough. However, for the state-of-the-art comparison reported in Table VI we set $W_{\text {anom }}=30$, as this value maximises the F1 score.

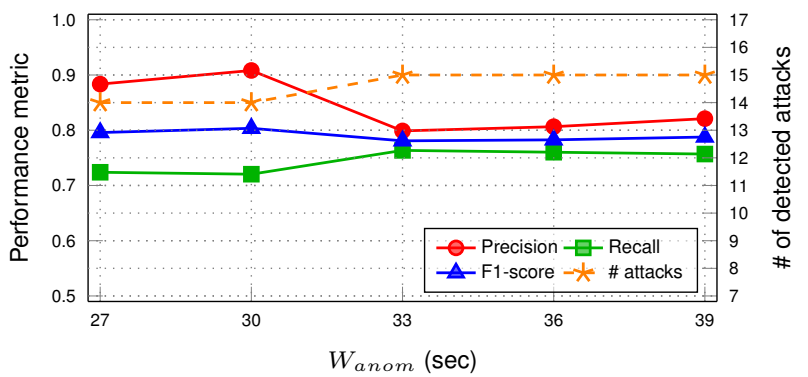

Figure 7: Sensitivity of WDNN to $W_{\text {anom }}$ on WADI dataset.

False alarms. With the settings listed in Table IV] DAICS produces 226 false positives on the WADI test set, equivalent to 5.3 human interventions per hour on average. As for the SWaT dataset, we measured the sensitivity of DAICS to the grace time $W_{\text {grace }}$. The results, reported in Figure 8 , show that increasing $W_{\text {grace }}$ reduces the number of intervention to 1.95 per hour on average at $W_{\text {grace }}=20$, although negatively impacting on precision, recall and F1 score even at $W_{\text {grace }}=5$.

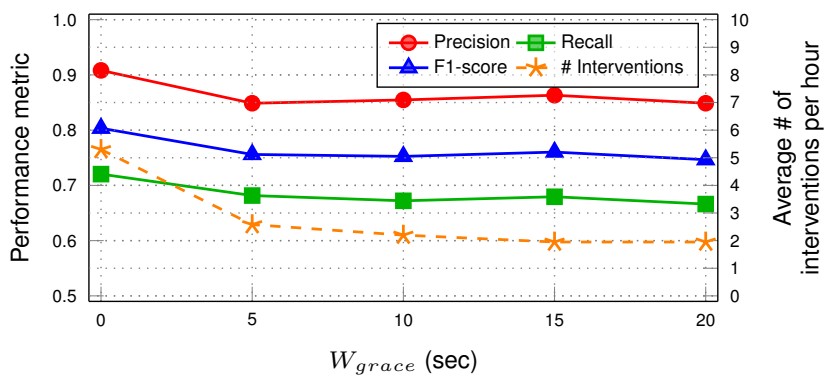

Figure 8: Sensitivity of WDNN to $W_{\text {grace }}$ on WADI dataset.

\section{E. Experiment 3: robustness to additive noise}

In this experiment, we measure the robustness of DAICS to noise added to the sensors readings. In our experiments, we add various levels synthetic noise to the sensor readings of both SWaT and WADI datasets. As anticipated in Section VII-B, we use white Gaussian noise with mean $\mu=0$ and increasing standard deviation $\sigma \in\{1,2,3,5,10,15\}$. We then observe the behaviour of DAICS with respect to the number of detected attacks and the detection accuracy measured with the F1 score metric. We then extend the experiment 2 in [19], where AADS and the CNN proposed in [20] are compared, by adding the performance of DAICS on the SWaT dataset.

Figures $9 \mathrm{a}$ and $9 \mathrm{~b}$ report on the performance of DAICS as a function of the Gaussian noise level. Although the trend of the point-based F1 score is similar on both datasets, we can observe a higher impact of the synthetic noise on the detection of attacks in the SWaT dataset. We recall that an attack is determined when the conditions in Equation 3 are met. In the case of sensors, the MSE must be above the threshold for $W_{\text {anom }}$ consecutive samples. However, the increasing number of point-based false negatives introduced with the noise, as 


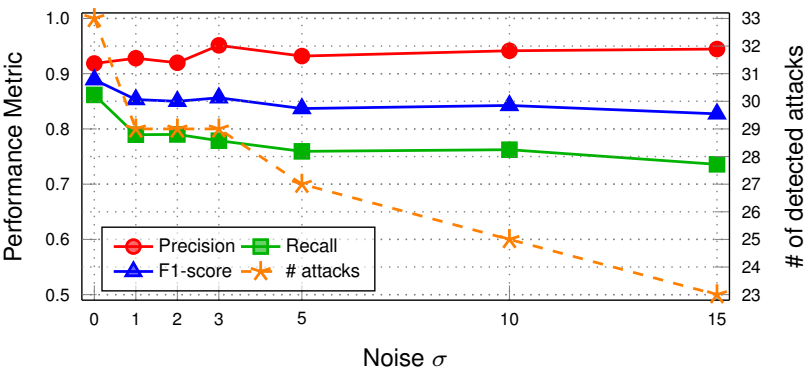

(a)

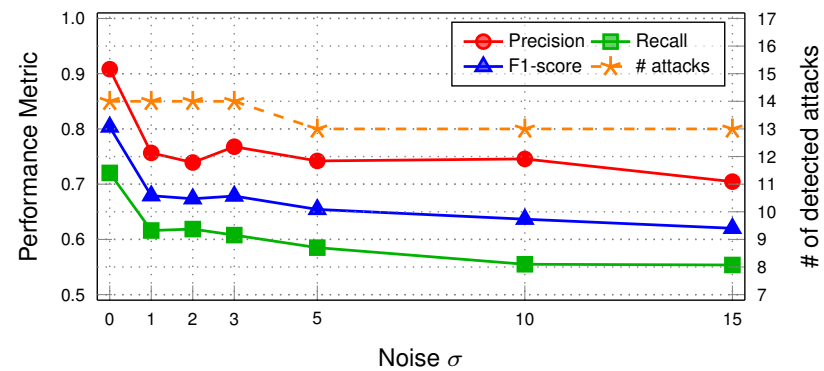

(b)

Figure 9: Performance of DAICS when adding Gaussian noise to the SWaT (a) and WADI (b) test sets.

shown in the two figures by the trend of the recall measure, reduces the chances of finding $W_{\text {anom }}$ consecutive point-based positive samples. Of course, this affects more the detection of short attacks, which are more frequent in the SWaT dataset.

Figure 10 shows the values of F1-score and number of detected attacks as functions of $\sigma$. We can notice that DAICS improves AADS in the number of detected attacks at any level of noise, except for $\sigma=15$ while almost matching the F1 score (we measured a slight decrease of 0.015 on average for $\sigma>0$ ). This demonstrates that the new mechanism is solid also in noisy conditions. On the contrary, the F1 score of the state of the art CNN drops drastically (green solid curve). This is mainly due to the statistical approach and to the static threshold employed to detect the anomalies, empirically selected as the value $T \in[1.8,3]$ that maximizes the F1-score. The low values of the F1-Score for $\sigma>0$ are due to a low precision measure (around 0.31 on average), meaning that the CNN classifies most of the records as anomalies, mostly false positives. This is the reason why the 36 attacks in the SWaT test set are almost all correctly classified (green dashed line). However, as everything looks like an anomaly, in a real-world deployment, the output of the CNN would be unusable.

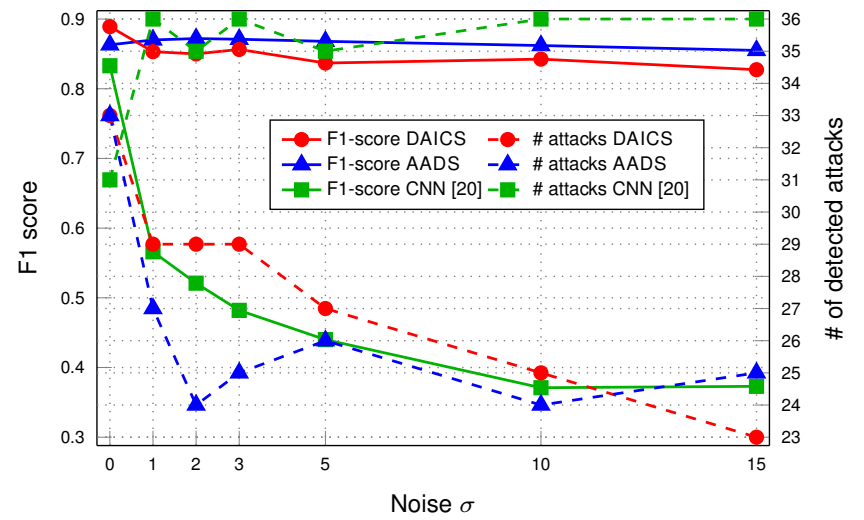

Figure 10: Performance when adding Gaussian noise to the SWaT test set.

In conclusion, DAICS overcomes state-of-the-art solutions on both SWaT and WADI datasets with respect to pointbased F1 score and number of detected attacks. In addition, DAICS is more robust to the additive noise, hence potentially more reliable in real-world industrial scenarios where the electromagnetic noise and the natural degradation of the devices might result in noisy data.

\section{CONCLUSION}

In this paper, we have presented DAICS, a framework for anomaly detection in Industrial Control Systems (ICSs) grounded on the one-class classification paradigm. DAICS has been designed to mitigate two major problems affecting similar solutions proposed in the state-of-the-art literature. The first, called domain shift, can be observed when changes in the normal behaviour of the ICS are not correctly handled by the detection system. The result is often an increase in the number of false alarms. The second problem is caused by the presence of noisy data, either due to interference on the communication channel within the ICS or to the ageing of devices, which prevents the detection system from correctly segregating the anomalies from normal operations.

We have tackled the aforementioned problems by introducing a fast and automated mechanism for updating the ICS model based on the detected false alarms caused by changes in the normal behaviour. Such a mechanism, based on the socalled few-time-steps algorithm, has been designed to be usable in production environments. Indeed, the only assumption is that the technician can identify the false alarms caused by maintenance operations (hardware/software updates) or known misbehaving devices, and to signal them to DAICS. This sets DAICS apart from similar solutions, where an in-depth knowledge of the underlying algorithms is necessary to update thresholds or other parameters. The combination of the fewtime-steps algorithm with a dynamic threshold mechanism, also proposed in this work, allows DAICS to overcome stateof-the-art solutions in terms of number of detected attacks and resilience to noisy data samples.

\section{ACKNOWLEDGMENT}

The authors would like to thank the Center for Research in Cyber Security at the Singapore University of Technology and Design for providing the SWaT and WADI datasets.

\section{REFERENCES}

[1] NCCIC/ICS-CERT, "Cyber-attack against ukrainian critical infrastructure," 2016. [Online]. Available: https://ics-cert.us-cert.gov/ alerts/IR-ALERT-H-16-056-01 
[2] S. Karnouskos, "Stuxnet worm impact on industrial cyber-physical system security" in IECON 2011-37th Annual Conference of the IEEE Industrial Electronics Society. IEEE, 2011, pp. 4490-4494.

[3] Q. Lin, S. Adepu, S. Verwer, and A. Mathur, "Tabor: A graphical modelbased approach for anomaly detection in industrial control systems," in Proc. of the 2018 on Asia Conference on Computer and Communications Security, 2018.

[4] M. Kravchik and A. Shabtai, "Efficient cyber attack detection in industrial control systems using lightweight neural networks and pca," Preprint arXiv:1907.01216, 2019.

[5] J. Goh, S. Adepu, M. Tan, and Z. S. Lee, "Anomaly detection in cyber physical systems using recurrent neural networks," in Proc. of the IEEE 18th International Symposium on High Assurance Systems Engineering (HASE), 2017.

[6] D. Shalyga, P. Filonov, and A. Lavrentyev, "Anomaly detection for water treatment system based on neural network with automatic architecture optimization," arXiv preprint arXiv:1807.07282, 2018.

[7] H. Cheng, et al., "Wide \& deep learning for recommender systems," in Proc. of the 1st workshop on deep learning for recommender systems. ACM, 2016, pp. 7-10.

[8] Y. L. Cun, B. Boser, J. S. Denker, R. E. Howard, W. Habbard, L. D Jackel, and D. Henderson, Handwritten Digit Recognition with a BackPropagation Network. Morgan Kaufmann Publishers Inc., 1990, p. 396404.

[9] S. Potluri, S. Ahmed, and C. Diedrich, "Convolutional neural networks for multi-class intrusion detection system," in Proc. of International Conference on Mining Intelligence and Knowledge Exploration, 2018.

[10] R. Doriguzzi-Corin, S. Millar, S. Scott-Hayward, J. Martnez-del-Rincn, and D. Siracusa, "Lucid: A Practical, Lightweight Deep Learning Solution for DDoS Attack Detection," IEEE Transactions on Network and Service Management, vol. 17, no. 2, pp. 876-889, 2020.

[11] M. T. Nguyen and K. Kim, "Genetic convolutional neural network for intrusion detection systems," Future Generation Computer Systems, vol. 113 , pp. $418-427,2020$.

[12] S. Potluri, S. Ahmed, and C. Diedrich, Securing Industrial Control Systems from False Data Injection Attacks with Convolutional Neural Networks, 2020, pp. 197-222.

[13] H. Yang, L. Cheng, and M. C. Chuah, "Deep-Learning-Based Network Intrusion Detection for SCADA Systems," in Proc. of IEEE Conference on Communications and Network Security (CNS), 2019.

[14] C. Chang, W. Hsu, and I. Liao, "Anomaly Detection for Industrial Control Systems Using K-Means and Convolutional Autoencoder," in Proc. of International Conference on Software, Telecommunications and Computer Networks (SoftCOM), 2019.

[15] C. Finn, P. Abbeel, and S. Levine, "Model-agnostic meta-learning for fast adaptation of deep networks," arXiv preprint arXiv:1703.03400, 2017.

[16] A. Nichol, J. Achiam, and J. Schulman, "On first-order meta-learning algorithms," arXiv preprint arXiv:1803.02999, 2018.

[17] iTrust, "iTrust Datasets." [Online]. Available: https://itrust.sutd.edu.sg/ testbeds/secure-water-treatment-swat

[18] S. Adepu and A. Mathur, "Generalized attacker and attack models for cyber physical systems," in Proc. of 40th Annual Computer Software and Applications Conference (COMPSAC), 2016.

[19] M. Abdelaty, R. Doriguzzi-Corin, and D. Siracusa, "AADS: A noiserobust anomaly detection framework for industrial control systems," in Proc. of the 2019 International Conference on Information and Communications Security (ICICS), 2019.

[20] M. Kravchik and A. Shabtai, "Detecting cyber attacks in industrial control systems using convolutional neural networks," in Proc. of the 2018 Workshop on Cyber-Physical Systems Security and PrivaCy, 2018

[21] H. Farsi, A. Fanian, and Z. Taghiyarrenani, "A novel online state-based anomaly detection system for process control networks," International Journal of Critical Infrastructure Protection, vol. 27, p. 100323, 2019.

[22] M. Q. Ali, E. Al-Shaer, H. Khan, and S. A. Khayam, "Automated anomaly detector adaptation using adaptive threshold tuning," ACM Transactions on Information and System Security, vol. 15, no. 4, pp. 1-30, 2013.

[23] D. Park, Y. Hoshi, and C. C. Kemp, "A multimodal anomaly detector for robot-assisted feeding using an lstm-based variational autoencoder," IEEE Robotics and Automation Letters, vol. 3, no. 3, pp. 1544-1551, 2018.

[24] J. Goh, S. Adepu, K. N. Junejo, and A. Mathur, "A dataset to support research in the design of secure water treatment systems," in Proc. of International Conference on Critical Information Infrastructures Security, 2016.

[25] S. Adepu and A. Mathur, "An investigation into the response of a water treatment system to cyber attacks," in Proc. of IEEE 17th International Symposium on High Assurance Systems Engineering (HASE), 2016.
[26] C. M. Ahmed, V. R. Palleti, and A. P. Mathur, "Wadi: a water distribution testbed for research in the design of secure cyber physical systems," in Proc. of the 3rd International Workshop on Cyber-Physical Systems for Smart Water Networks, 2017

[27] J. Quionero-Candela, M. Sugiyama, A. Schwaighofer, and N. D. Lawrence, Dataset shift in machine learning. The MIT Press, 2009.

[28] M. Wang and W. Deng, "Deep visual domain adaptation: A survey," Neurocomputing, vol. 312, pp. 135-153, 2018.

[29] P. Mulinka and P. Casas, "Adaptive network security through stream machine learning," in Proc. of the ACM SIGCOMM 2018 Conference on Posters and Demos, 2018.

[30] P. Mulinka and P. Casas, "Stream-based machine learning for network security and anomaly detection," in Proc. of the 2018 Workshop on Big Data Analytics and Machine Learning for Data Communication Networks, 2018

[31] W.-Y. Chen, Y.-C. Liu, Z. Kira, Y.-C. F. Wang, and J.-B. Huang, "A closer look at few-shot classification," Preprint arXiv:1904.04232, 2019.

[32] I. Sutskever, J. Martens, G. Dahl, and G. Hinton, "On the importance of initialization and momentum in deep learning," in International conference on machine learning, 2013, pp. 1139-1147.

[33] G. C. Goodwin, D. E. Quevedo, and E. I. Silva, "Architectures and coder design for networked control systems," Automatica, vol. 44, no. 1, pp. 248-257, 2008.

[34] X.-S. Zhan, Z.-H. Guan, F.-S. Yuan, and X.-H. Zhang, "Performance analysis of networked control systems with snr constraint," International Journal of Innovative Computing, Information and Control, vol. 8, no. 12, pp. 8287-8298, 2012.

[35] A. Paszke, S. Gross, F. Massa, A. Lerer, J. Bradbury, G. Chanan, T. Killeen, Z. Lin, N. Gimelshein, L. Antiga et al., "Pytorch: An imperative style, high-performance deep learning library," in Advances in neural information processing systems, 2019, pp. 8026-8037.

[36] G. M. Kurtzer, V. Sochat, and M. W. Bauer, "Singularity: Scientific containers for mobility of compute," PLOS ONE, 2017.

[37] ODVA, "Technology overview series: Ethernet/ip," Tech. Rep., 2016.

[38] B. Galloway and G. P. Hancke, "Introduction to industrial control networks," IEEE Communications surveys \& tutorials, vol. 15, no. 2, pp $860-880,2012$

[39] D. Li, D. Chen, B. Jin, L. Shi, J. Goh, and S.-K. Ng, "Mad-gan: Multivariate anomaly detection for time series data with generative adversarial networks," in International Conference on Artificial Neural Networks. Springer, 2019, pp. 703-716. 